\title{
Impact of carrying capacity on the functioning of the railway network
}

\author{
Alexey Volodin ${ }^{1, *}$, Nadezhda Gudkova ${ }^{1}$, Alexey Astafiev ${ }^{2}$, Vadim Alferov $^{1}$, and Jozef \\ Majerčák ${ }^{3}$ \\ ${ }^{1}$ Department of Operation of Water Transport, Russian University of Transport, Moscow, \\ Obraztsova str., 9, building 9, 127994. Russia. Email: ab.volodin@mail.ru \\ ${ }^{2}$ Department of Transport business management and intelligent systems, Russian University \\ of Transport, Moscow, Obraztsova str., 9, building 9, 127994. Russia. Email: aleks- \\ astaf@yandex.ru \\ ${ }^{3}$ Faculty of Operation and Economics of Transport and Communications, Zilina, Univerzitná \\ 8215/1, 010 26, Republic of Slovakia. Email: jozef.majercak@fpedas.uniza.sk
}

\begin{abstract}
Traffic flows associated with the movement of passengers and goods, characterized by traffic intensity, speed, direction and other factors are closely interrelated. The planned traffic intensity of these flows is determined by calculating the carrying capacity of the elements of the transport system or links in the delivery chains. The article provides a comprehensive analysis of the throughput capacity of a railway line, railway station, railway stations and landside areas, airports, and highways. Calculated dependencies, used to determine the carrying capacity of transport infrastructure facilities, have been carry out. It is shown that the throughput (capacity) of infrastructure facilities is focused, most of all, on the volume of cargo transportation in various industries. The factors that must be take into account when determining the conditions for further development and increasing the capacity of transport infrastructure elements are noted. All calculation methods are reduced to determining the maximum permissible volume of cargo transportation (in passenger traffic, passengers) that can be passed (processed) by an infrastructure object per unit (for a certain period) of time in the considered road transport and weather and climatic conditions.
\end{abstract}

\section{Introduction}

A well-developed system of transport communications stimulates the mobility of the population, which in turn leads to an equalization of the rates of economic growth and investment attractiveness of various regions that are connected with each other. At the same time, the cultural and educational level of society is increasing [1].

The throughput capacity of a transport infrastructure object is understood as the maximum permissible number of trains, cars, etc., which can be passed (processed) by this object for a specific period of time (day, hour, etc.) the existing (planned, projected) technical equipment, the established number and placement of personnel, subject to the requirements of the Technical Operation Rules for the appropriate type of transport and other regulatory technical acts. 
Throughput capacity is influence by many factors depending on the technical and technological parameters of the infrastructure. Therefore, in order to obtain reliable data, such indicators taken into account, which characterize the interaction of reloadable objects and infrastructure elements in various conditions $[2,3]$.

If we consider all the variety of objects in the transport sector, then we can single out a number of objects for which Throughput capacity will be one of the main indicators and conditions of operation - these are railway lines, stations, railway terminals (freight, customs, cash offices, etc.), sea and river ports, berths, airports, transport hubs, pipelines (oil, gas, product pipelines), etc.

\section{Materials and Methods}

The problems of a developed system of transport communications are devoted to the works of such authors as Gasparik J., Kudlac, S., Kurenkov P., Majercak, J., Pokrovskaya O. Increasing the requirements for optimizing the capacity of transport infrastructure facilities is the most important task for the socio-economic development of regions and municipalities of the state.

For this reason, the purpose of the study is to conduct a comprehensive economic analysis of the capacity of transport infrastructure facilities.

During the research, the following research methods used: expert assessments, calculation and analytical methods, comparison method, statistical and quantitative methods, survey methods.

The research carried out in the following stages:

1. Calculation of the capacity of the railway line.

2. Calculation of the capacity of the railway station.

3. Calculation of the throughput of stations and landside areas.

4. Calculation of airport capacity.

5. Calculation of the capacity of highways.

6. Development of measures for the development of transport infrastructure in the identified problem areas.

During the study, the following research methods used system analysis, calculation and analytical methods, comparison method, methods of mathematical modeling, statistical and quantitative methods.

\section{Results}

The cash throughput of a railway section is the maximum number of trains (pairs of trains) that can be pass along the railway section per day, depending on its technical equipment, traction power, type of traffic schedule and technology of organizing train traffic [4].

The capacity depends on the type of train schedule, the value of key indicators, as well as on the track development of separate points and is determined by the formula [5]:

$$
N=\frac{\left(1440-t_{t e c h}\right) \alpha_{r e l}}{T_{\min }}
$$

where

$t_{\text {tech }}$ - duration of technological "windows", min;

$\alpha_{\text {rel }}$ - coefficient of reliability of technical means;

$T_{\text {min }}$ - chart period, min 
We calculate the available throughput of the site in the following sequence:

1) the maximum distance on the section with the greatest sum of the running times of even and odd trains is determined;

2) 4 possible schemes for the laying of trains are consider on the established stretch and a scheme is select that provides the minimum period of the schedule;

3) a scheme is drawn up for laying trains on adjacent hauls, stops are regulated in even and odd directions.

The required throughput of the railway. Lines - this is the largest number of trains that must be passed with a parallel schedule and given dimensions of movement of passenger, suburban, group trains, the influence of which on the throughput is taken into account through the take-off rates, depending on the track development of separate points, their number, speed and the location on the graph of passenger suburban, express, freight and groupie trains [6].

The required bandwidth is determine by the formula:

$N_{\text {req }}=N_{\text {acc.fre.tr }} \cdot \beta_{\text {res }}+\varepsilon_{\text {pas }} \cdot N_{\text {pas }}+\varepsilon_{\text {sub }} \cdot N_{\text {sub }}+\left(\varepsilon_{\text {exp }}-1\right) \cdot N_{\text {exp }}+\left(\varepsilon_{g r}-1\right) \cdot N_{g r}$,

where,

$N_{\text {acc.frb.tr }}-$ the size of the movement without taking into account groupie and accelerated freight trains;

$N_{\text {pas }}, N_{\text {sub }}, N_{\text {exp }}, N_{g r}$ - sizes of passenger, suburban, express freight and groupie trains, respectively;

$\varepsilon_{\mathrm{pas}}, \varepsilon_{\mathrm{sub}}, \varepsilon_{\mathrm{exp}}, \varepsilon_{\mathrm{gr}}-$ pickup rates of freight trains by passenger, suburban, express freight and groupie trains, respectively;

$\beta_{\text {res }}-$ reserve ratio.

On a single-track section, the take-off ratio for all categories of trains, except for combined trains, is determine by the formula:

$$
\varepsilon=1+0,6 \cdot \gamma_{\text {pack }}-\frac{\left(20 \cdot \mathrm{C}_{4}^{r}\right)}{N_{\text {tot }}^{\text {f.s.e }}} \geq 1,
$$

where,

$N_{\text {tot }}^{f . s . e}$ - the total number of fast, passenger, suburban and express trains on the site;

$\mathrm{C}_{4}^{r}$ - is the proportion of four-track separate points (four or more tracks, including the main one) on the calculated section, this is the ratio of the number of four-track separate points to the total number of separate points on the section;

$\gamma_{\text {пак }}$ - pack - batch factor, which is determined by the formula:

$$
\gamma_{\text {пак }}=\frac{N_{\text {pack }}}{N}
$$

where,

$N_{\text {pack }}$ - the number of train pairs in the schedule period, passed in packets;

$\mathrm{N}$ - is the total number of train pairs in the schedule period.

For a double-track section, the removal rate is:

$$
\varepsilon=\left(t_{f . t r}\left(1-\Delta_{n . a v}\right) \cdot\left(0,8-0,005 \cdot N_{\text {tot }}^{f . s . e}\right) / I\right)+1,3
$$

where,

$t_{f . t r}-$ travel time of a freight train along a limiting haul, min;

$\Delta_{n . a v}$ - is the ratio of the net average travel time of a pair of passenger trains (fast, suburban, accelerated freight) to the net travel time of a pair of freight trains on the section: 


$$
\Delta_{n . a v}=\frac{t_{\text {pass.n.av }}^{\prime}+t_{\text {pass.n.av }}^{\prime \prime}}{t_{\text {fr.n.av }}^{\prime}+t_{\text {fr.nav }}^{\prime \prime}}
$$

Separately, for group trains on a single-track line, the removal rate is calculate as:

$$
\varepsilon_{c . t r}=\delta \cdot\left(1,2+0,9 \cdot \mathrm{C}_{c . t r}\right)-0,4 \cdot N_{\text {tot }}^{f . s . e} \cdot\left(1-\Delta_{\text {n.av }}\right)-0,5 \geq 1,
$$

$\delta$ - is the ratio of the sum of the inter-train intervals in the odd and even directions of movement to the period of the non-batch parallel schedule on the limiting stretch;

$C_{c . t r}$ - the number of stations on the section, served by the combined train.

On a double-track line for group trains $\varepsilon_{c . t r}$ is equal to:

$$
\varepsilon_{c . t r}=\left(\mathrm{C}_{\text {c.tr }}+1\right) \cdot\left[1-0,02 \cdot N_{\text {tot }}^{f . s . e} \cdot\left(2-\Delta_{\text {n.av }}\right)\right] \geq 1
$$

Since the definition of throughput for this calculation is general, it does not take into account the actual operating conditions that may be encounter on the entire railway network of Russian Railways.

Therefore, for example, the problems of "congestion" at the approaches to seaports lead to a critical situation in the movement of trains and the appearance of "abandoned" trains along the entire route [7]. In addition, with a decrease in traffic volumes, the nature and type of stations changes - marshalling stations are transferor to the category of divisional ones, and divisional ones - to the category of intermediate ones. Many stations are shutting down altogether, as are some individual lines, resulting in reduced throughput. However, at present there is a question about the restoration of the lines, which closed for the movement $[8,9]$.

The need to save costs for the maintenance of permanent devices that do not depend on the size of traffic is the reason for the conservation of station tracks, station parks, odd or even sorting systems, etc. Because of this, many railroads. Stations are transferor from daily to semi-daily operation, because of which the class of stations decreases.

In the existing methods for determining the throughput, the elements of the railway lines are considered separately, without taking into account specific conditions, including without taking into account the possibility of congestion at the approaches to seaports and places of mass unloading, since the process of transportation of goods is multiphase in nature.

The carrying capacity of the railway stations - the maximum possible number of freight (without processing and with processing separately) and passenger trains that can be passed by the station per day, taking into account all adjacent directions, depending on the technical equipment of the station with full use of available funds. Determined by the capacity for station tracks for the reception and departure of trains and for switch necks [10].

The available capacity of any station established only if it not limited by the capacity of adjacent devices.

The value of the available throughput of station tracks depends on the duration of the track occupation by trains, the number of tracks in the calculated fleet, the movement of passenger trains on the approaches to the station, the performance of work on the current maintenance and scheduled repair of the track and contact network and other factors [11].

The design of the neck, the method of controlling the switches, the duration of various movements, the possibility of sectional opening and the use of switches, failures of technical means and the time for their maintenance and scheduled repair have an impact on the available throughput of the switch throat.

Thus, the capacity of the station determined by the following formula:

$$
N=\gamma \cdot\left(1440-T_{\text {d.op }}\right) \cdot \alpha_{\text {rel }} \cdot\left[\frac{\beta_{l e n} \cdot m^{*}}{t_{\text {av.t }}+\Delta t}+\frac{\left(1-\beta_{l e n}\right) \cdot m}{t_{\text {av.t }}}\right]
$$


$m$ - is the number of tracks in the park or at the station;

$T_{\text {d.op- }}$ duration of operations to maintain permanent devices, min;

$t_{\text {av. }}$ - $^{-}$verage time of occupation of a path by a train, min;

$\alpha_{\text {rel }}$ - reliability factor;

$m^{*}$ - the number of long paths, including paths with the occupation of necks and turnout sections;

$\beta_{\text {len }}$ - is the share of trains of the scheduled length in the total flow;

$\Delta \mathrm{t}$ - additional time of occupying tracks for stretching the "head" of the train or upsetting the "tail" when receiving and sending long-train trains in one section;

$\gamma$ - is the efficiency factor of using the train schedule for their organized supply to the junction or technical station, depending on the limitation of the inter-train interval for the power supply systems, the availability of sufficient capacity tracks at the pre-junction intermediate stations, to change the order of trains and other factors [12].

In the formulas for calculating the cash and required throughput of the railway lines and railway stations, the reliability of technical and permanent devices (formulas (1) and (8)) is taken into account, but the irregularity associated with the irregularity of the departure of trains from loading stations, as well as with the lack of synchronization of the movement of freight, carriage and train flows, is not taken into account. Also, the configuration and density of the network, the saturation of specific sections, directions and polygons with moving trains, and "abandoned" trains with laden and empty wagons, both in the process of transportation and temporarily set aside from traffic and waiting for "lifting" .

The main elements of the transport system of any constituent entity of the Russian Federation are railway, bus and river stations. Ensuring the effective use of various types of transport is due to the correct location of the station complexes and their throughput.

The station is part of the railway. a complex or any other station complex, passenger areas of ports, central bus terminals, which contain all interconnected buildings, structures and devices designed to serve passengers, perform postal, baggage and other operations.

The throughput of the stopping point of the city transport at the station square can be calculate using the formula:

$$
N=\frac{3600}{T_{d . p}+\tau+r}
$$

where,

$\mathrm{N}$ - is the number of vehicles that can be passed through the stopping point per hour in a certain direction, units;

$\mathrm{T}_{\text {d.p }}$ - average duration of parking during embarkation and disembarkation of passengers, $\mathrm{s}$; $\tau$ - is the time spent on maneuvers and approaching the stopping point, s;

$r$ - time spent on acceleration and deceleration of transport, $s$.

If we accept for further calculation:

$$
T=T_{d . p}+\tau+r
$$

then the total throughput of the station area N_pl will be determined by the formula:

$$
N_{p l}=m \frac{3600}{T} K
$$

From formula (11) it can be seen that the capacity of the station area depends on the number of stopping points $\mathrm{m}$, taking into account the delays due to non-simultaneous departures of urban transport from stopping points by the coefficient K [13]. 
Airport - a complex of devices and structures that are intended for the reception, departure, placement of aircraft and maintenance of air traffic, which has an airfield, an air terminal, cargo terminals and other facilities and equipment to perform these operations.

The capacity of an airport is understood as the maximum possible volume of passenger $W_{\text {pass }}$ and postal-cargo $W_{c}$ traffic that can be performed through a given airport with the available technical means of providing flights and ground handling of aircraft and helicopters, the established number and placement of personnel, subject to the requirements and norms and standards of aviation and transport safety.

The airport's capacity by the number of passengers carried per year is determine by the formula:

$$
W_{\text {pass }}=q \cdot \lambda_{h}^{\max } \cdot \frac{T_{h} \cdot 365}{k_{d} \cdot k_{h}}
$$

where,

$q$ - average planned number of passengers per plane;

$\lambda_{h}^{\max }$ - the capacity of the aerodrome;

$T_{h}$ - the number of hours of airport operation per day.

Analyzing formula (12), we can conclude that when calculating the airport capacity, the coefficients of not only the daily irregularity $k_{d}$, but also the hourly irregularity $k_{h}$ of aircraft movement are taken into account, which show the ratio of the maximum daily number of takeoff and landing operations to the daily average per year and the ratio of the maximum hourly the number of takeoff and landing operations to the hourly average for the maximum day, respectively (see formula (12)).

The average planned number of passengers per plane is calculate as:

$$
q=S \cdot p_{i} \cdot m_{i} \cdot j_{i},
$$

$p_{i}$ - the ratio of the frequency of movement of aircraft of different types;

$m_{i}$ - aircraft passenger capacity;

$j_{i}$ - coefficient taking into account the percentage of commercial load (according to statistics $\left.j_{i}=0,75\right)$.

Predicting $p_{i}$ and $m_{i}$ for several years ahead is not an easy task, so you should determine the value of $q$ based on statistical data for a certain number of years.

The runway capacity is the maximum possible number of takeoff and landing operations of aircraft and helicopters per unit (for a specified period) of time, which is provide at a given aerodrome in compliance with aviation and transport safety requirements.

The runway throughput is influenced by the number and placement of connecting lanes, the layout of the runways, the types of aircraft operated and their flight modes, local conditions, the equipment of airfields with landing aids, the organization of aircraft traffic at the airfield, airfield. The throughput of the runway determined by the following formula:

$$
\Pi_{\text {theor }}=\frac{60}{\Delta T}
$$

where,

$\Delta \mathrm{T}$ is the average duration of takeoff and landing operations.

Road throughput is the maximum number of cars that a road section can pass per unit (for a set period) of time in one or two directions with known design parameters of this road (lane, superstructure structure, type of coverage, maximum permissible speed, etc.) and given weather and climatic conditions.

A distinction made between theoretical, practical and calculated bandwidth. The theoretical throughput $P_{\mathrm{T}}$ is calculate for a horizontal road section, taking into account that the intervals between cars and a homogeneous composition of the traffic flow (for example, only cars of a passenger type) are constant. The theoretical throughput of the highway lane 
is about 2,900 "cars" per hour. The practical throughput is determined in the real traffic conditions on the roads.

The estimated throughput characterizes the economically feasible number of cars that can pass a section per unit of time in the considered road conditions in accordance with the adopted traffic management scheme.

When assessing the practical throughput under specific road conditions, it is necessary to take into account the decrease in throughput due to road conditions by the $\beta$ coefficient, and the power of the roadway found using the following formula, taking into account the maximum practical throughput of cars per hour $P_{\text {max }}$ in the calculation:

$$
P=\beta \cdot P_{\max },[\text { car./h. }]
$$

For automobile lines, as well as for railways, also, the unevenness of traffic associated with the irregularity of the departure of cars and from the starting points taken into account.

The maximum practical throughput $P_{\max }$ takes into account the density of the network and its configuration, the saturation of various types of cars in certain areas (table 1).

Table 1. Values of the maximum practical road throughput

\begin{tabular}{|l|c|}
\hline \multicolumn{1}{|c|}{ Roads } & $\boldsymbol{P}_{\boldsymbol{m a x}}$, , cars / h \\
\hline Two-lane & 3600 in both directions \\
\hline Three-lane & 4000 in both directions \\
\hline Four-way: & 2100 one lane each \\
\hline no dividing line & 2200 one strip each \\
\hline with a dividing strip & \\
\hline Six-lane: & 2200 one lane each \\
\hline no dividing line & 2300 one strip each \\
\hline with a dividing strip & 2300 one lane each \\
\hline Highways with eight lanes & \\
\hline
\end{tabular}

The maximum practical throughput is set at the control site under favorable weather and climatic conditions and traffic, consisting only of passenger cars [14].

\section{Discussion}

Thus, the existing approaches to determining the throughput of transport infrastructure facilities take into account many indicators and conditions of interaction between moving transport, freight and passenger flows with infrastructure elements in various functioning models, show how efficiently a certain element functions, and also identify "narrow »Places that impede the rhythm and uninterrupted functioning of the entire transport system.

However, all calculation methods are reduce only to determining the maximum number of transportation objects (vehicles, cargo and passengers) that a particular infrastructure element can skip per unit of time.

The existing methods for determining the throughput do not take into account the configuration of communication routes, the trajectories of transport, cargo and passenger flows along them, the irregularity of the dispatch of goods and passengers from the places of origin of the corresponding flows, the asynchrony of their movement along parallel or sequentially located polygons and sections, as well as others factors.

These factors must be taken into account as initial data to determine the conditions for further development and increase the capacity of transport infrastructure elements, or, con- 
versely, to consider the feasibility and possibility of their temporary closure (conservation), as well as partial or complete dismantling in order to reduce their costs content.

\section{References}

1. Elect. fund of leg. \& regul. tech. doc. / ODM 218.2.020-2012 Method. recom. for asses. the throughput of roads. - Access mode: http://docs.cntd.ru/document/1200092512.

2. P. Kurenkov, O. Pokrovskaya, M. Anastasov, M. Sokolov, A. Bochkov. Study of the current state of the transport infrastructure of road and rail transport of the Russian Federation, in IOP Conference Series: Materials Science and Engineering, 1-5 October 2019, Kislovodsk, Russia, 698, 6 (2019)

3. M. Babin, M. Buda, J. Majercak. Terminals for Transportation of Dangerous Goods, in Proceedings of $16^{\text {th }}$, International Conference on Transport. 25-26 October 2012, Kaunas, Lithuania (2012)

4. Recom. for the design of stations. - M: Cent. Res. Instit. of Urban Planning, (1997)

5. Hoare Ch. Interacting sequential processes. Mir (1989)

6. L. Ivankova, A. Burakova. Determination of the throughput of stations taking into account the capacity of track development, in Proceedings Modern technologies. System analysis (2018)

7. S. Miloslavskaya, E. Plotnikova. Current situation and optimization of inland waterway infrastructure financing. in Proceedings Transport Problems, 13, 3 (2018)

8. J. Rodrigue. The Geography of Transport Systems. London and New York: Taylor \& Francis (2006)

9. E. Majercakova, P. Majercak, J. Majercak. Logistics and New Trends in Supply Chain Management in Times of Economic Crisis, in the 3rd International Conference on Education Reform and Management Innovation, ERMI, 05-06 September 2015, Bangkok, Thailand, 78 (2015)

10. Z. Mejokh, N. Kapustina, D. Kakhrimanova, A. Safronova, A. Yussuf. Urban Transport and Logistics Infrastructure as an Element of Economic Security in the Region, in Advances in Intelligent Systems and Computing, 22-27 May 2020, Novosibirsk, Russia (2020).

11. A. Egorov, N. Pilipchuk, I. Khmelev, V. Shatokhin, A. Kovkin. World experience in the development of container traffic, in IOP Conference Series: Materials Science and Engineering, 1-5 October 2019, Kislovodsk, Russia (2019)

12. S. Kudlac, V. Stefancova, J. Majercak. Using the Saaty Method and the FMEA Method for Evaluation of Constraints in Logistics Chain, in 10th International Scientific Conference on Transportation Science and Technology, 04-05 May 2017, Vilnius, Lithuania (2017)

13. J. Majerčák, P. Majerčák. The impact of the dynamics of a train ride in a curve and its effect on a superstructure, in Transportation Research Procedia, 29-31 May 2019, Novy Smokovec, 40, (2019)

14. J. Gasparik, V. Luptak, P. Mesko, P. Kurenkov. Methodology for assessing transport connections on the integrated transport network. Komunikacie, 19, 2 (2017) 